\title{
Quantum Dot Blueing and Blinking Enables Fluorescence Nanoscopy
}

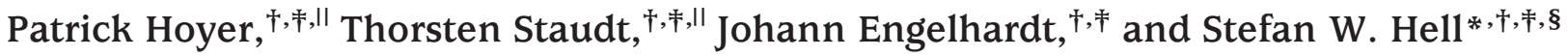 \\ ${ }^{\dagger}$ German Cancer Research Center (DKFZ), Optical Nanoscopy Division, Im Neuenheimer Feld 280, 69120 \\ Heidelberg, Germany, ${ }^{\ddagger}$ BioQuant Center, Im Neuenheimer Feld 267, 69120 Heidelberg, Germany, and ${ }^{\S}$ Max Planck \\ Institute for Biophysical Chemistry, Department of NanoBiophotonics, Am Fassberg 11, 37077 Göttingen, Germany
}

ABSTRACT We demonstrate superresolution fluorescence imaging of cells using bioconjugated CdSe/ZnS quantum dot markers. Fluorescence blueing of quantum dot cores facilitates separation of blinking markers residing closer than the diffraction barrier. The high number of successively emitted photons enables ground state depletion microscopy followed by individual marker return with a resolving power of the size of a single dot $(\sim 12 \mathrm{~nm})$. Nanoscale imaging is feasible with a simple webcam.

KEYWORDS Fluorescence microscopy, subdiffraction, resolution, stochastic, quantum dots, blinking

$\mathrm{F}$ or many years it was widely accepted that the resolution of any lens-based light microscope is limited to about half the wavelength of light. ${ }^{1}$ This notion has been changed by the discovery that fluorophore transitions between a fluorescent ("on") state and a nonfluorescent ("off") state, affords far-field optical imaging with diffractionunlimited spatial resolution. ${ }^{2,3}$ Specifically, serially enabling (groups of) markers to occupy the fluorescent on state, while ensuring that their surrounding neighbors remain in the off state, allowed the successive recording of features closer than the diffraction limit. Various far-field fluorescence nanoscopy techniques sharing this characteristic have emerged since then. ${ }^{4-7}$ They can be classified into two families depending on the way the switching and signal readout is implemented. ${ }^{7}$

In the targeted switching and readout approach with stimulated emission depletion (STED) and RESOLFT microscopy as its prototype, ${ }^{2,7}$ the on-off state contrast is prepared at a predefined coordinate at the sample using a light distribution featuring one or many zero intensity points or lines. The more recent stochastic approaches called (fluorescence) photoactivatable localization (PALM) $)^{4,6}$ and stochastic optical reconstruction (STORM) ${ }^{5}$ microscopy differ by the fact that the switching and readout are performed stochastically in space. In these concepts, wide-field illumination turns individual off state markers into the on state such that within the diffraction range $(<300 \mathrm{~nm})$ less than a single marker is able to emit on average. The coordinate of this marker is found out from calculating the centroid of its diffraction pattern rendered by a high aperture lens on a camera, which is possible if in its on state the marker emits $m$ photons. ${ }^{4-6}$ The position of the marker is

\footnotetext{
* To whom correspondence should be addressed, shell@gwdg.de.

"P.H. and T.S. are equal contributors.

Received for review: 10/17/2010

Published on Web: 12/03/2010
}

established with an accuracy of approximately $\Delta / \mathrm{m}^{1 / 2}$, where $\Delta$ denotes the full width at half-maximum (fwhm) of the diffraction spots and $m$ is the number of detected photons. ${ }^{8,9}$

The apparent need for photoactivatable or photoswitchable compounds, such as cyanines or switchable proteins, limited the general applicability of the actual PALM/STORM imaging. A solution to this problem was brought about by the insight that a stochastic type of nanoscopy can also be performed with elementary state transitions of conventional dyes, ${ }^{10}$ namely, through ground state depletion by shelving the dye in a metastable dark state. In ground state depletion microscopy followed by individual molecule return (GSDIM),${ }^{10}$ also called dSTORM, ${ }^{11}$ the ordinary singlet state of the fluorophore is the on state whereas a metastable dark state (e.g., a triplet or redox state) is its off complement. As these states are inherent to basically any fluorophore, GSDIM can operate with any fluorophores, provided the on-off rates are suitable. In GSDIM, excitation intensities are applied that switch all but $<1$ marker within the diffraction range to the metastable off state, from where individual markers stochastically return to the on state, which is the ordinary singlet system. The photon bursts from molecules in the singlet system are recorded before the molecules return to the off state or are bleached. Since fluorescence generation and off-switching is performed with a single laser wavelength, ${ }^{10,12}$ and because it requires just basic fluorophore states, GSDIM is the most straightforward far-field optical nanoscopy approach at present.

Therefore, the question arises as to whether GSDIM can be extended to inorganic nanocrystals. Fluorescent semiconductor quantum dots ${ }^{13}$ have received increased attention due to their remarkable photostability and brightness, ${ }^{14}$ both of which limit current nanoscopy methods. While a 4.4-fold increase in resolution over the diffraction barrier has been attained with special Mn-doped ZnSe nanocrystals in the RESOLFT mode by using excited-state absorption, ${ }^{15}$ this 


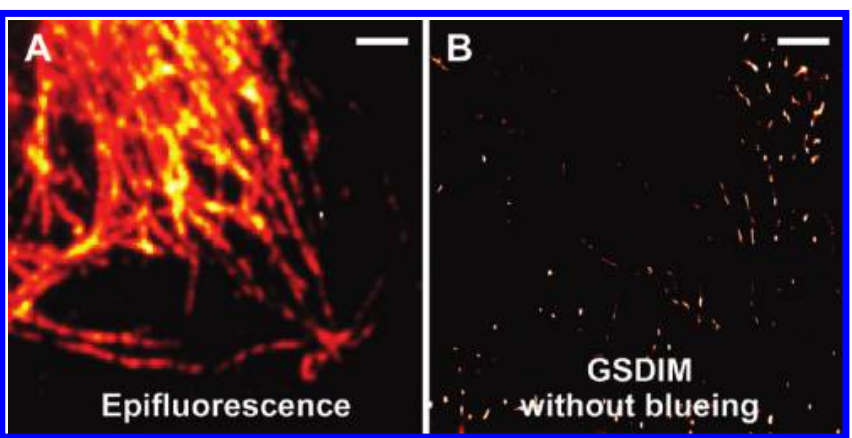

FIGURE 1. Blinking kinetics of native quantum dots limit their application in GSDIM. Epifluorescence (A) and GSDIM recording (B) of microtubules immunostained with quantum dots at typical concentrations. Due to short off times, signals elicited within subdiffraction distances overlap, causing localization to fail. Since the signal of these markers is discarded, structures appear interrupted. Scale bars: $2 \mu \mathrm{m}$.

on-off switching mechanism is not easily transferable to other quantum dot types.

A favorable attribute of quantum dots is that their photoluminescence may feature large intensity fluctuations, ${ }^{16}$ known as blinking. ${ }^{17}$ While recent quantum dot research has aimed at eliminating blinking, ${ }^{18-22}$ the stochastic toggling between an on and off state clearly bears potential for superresolution imaging. Therefore, quantum dot blinking has recently been used to enhance spatial resolution down to $50 \mathrm{~nm}$ in a technique called superresolution optical fluctuation imaging (SOFI) whereby the on-off fluctuations of the recorded frames are evaluated through correlation functions of different orders. ${ }^{23}$ In fact, blinking has been applied earlier to distinguish between two quantum dots within the diffraction limit in a technical sample. ${ }^{24}$ However, in order for a method to qualify as a superresolution imaging concept, it must be able to separate an, in principle, arbitrarily high number of markers, or at least a number of markers residing within the diffraction range that is as high as encountered in dense labeling. This goal has not been reached so far with quantum dots, because native quantum dots spent a rather short time in the off state, causing the diffraction spots from several emitters to overlap on the camera. With numerous localizations failing as a result, many markers have to be discarded, leaving dark patches in the images (Figure 1).

Since prebleaching or reducing the concentration of the marker is not satisfactory either, GSDIM of quantum dots requires observation of their blinking kinetics. The duration of the on-state periods can be easily controlled by the light intensity applied. The reason is that multiexciton generation and subsequent charging by Auger autoionization are elevated at high intensities, reducing the probability for long on-state periods during recording. ${ }^{25}$ The transition rate from an off to an on state, on the other hand, is ideally reduced to a level where all but one marker within the diffraction volume remain dark at a given point in time.
We now show that quantum dots indeed feature spectral properties suitable for far-field optical imaging beyond the diffraction barrier. Specifically, upon steady illumination, quantum dot emission continuously shifts toward shorter wavelengths, a phenomenon known as blueing. ${ }^{17,26,27}$ By stochastically affecting nearly all quantum dots of a densely labeled sample, blueing enables the sequential preparation of subclasses of quantum dots having different emission wavelengths within the diffraction volume. Exploiting the blinking of the transient subclass of blue-shifted quantum dots, GSDIM now yields images (of biological samples) with nanoscale resolution.

The process of gradual blueing is attributed to a continuous size reduction of the CdSecore due to photooxidation, ${ }^{17,27,28}$ thus differing from the minor effects of spectral diffusion and Stark shift caused by surface dipoles. ${ }^{29,30}$ Upon exposure to oxygen, selenium surface atoms of a CdSe core are photooxidized, creating an evaporating $\mathrm{SeO}_{2}$ surface film which causes the nanocrystal to degrade over time ${ }^{31}$ (Figure 2A). The increased confinement of excitons broadens the energetic gap between their valence and conduction bands and irreversibly blue shifts their emission wavelength. If not bleached earlier, the quantum dots are turned off by complete photooxidation of the core. The speed of which the emission peak shifts toward shorter wavelengths is proportional to the excitation intensity. The total spectral shift of the peak depends on the duration of the illumination.

As previously reported, CdSe/ZnS quantum dots exhibited an average blue shift of $29 \mathrm{~nm}$ from an initial emission wavelength of $570 \mathrm{~nm}$ under $20 \mathrm{~kW} \mathrm{~cm}{ }^{-2}$ excitation intensity before photobleaching. ${ }^{28}$ While higher laser intensities accelerate the process of blueing, ${ }^{32}$ slow spectral blue shifts have also been observed under low-level illumination from a Xe arc lamp $\left(\approx 3 \mathrm{~W} \mathrm{~cm}^{-2}\right){ }^{26}$ From our analysis of approximately 200 measured fluorescence time traces of individual photooxidized quantum dots, we deduced that the duration of the off periods remains unaffected by the blueing process (Figure 3). However, thanks to an individual time lag between initial illumination and the onset of blueing in each of the capped nanocrystals, ${ }^{27}$ the moment at which an individual core is photooxidized is randomly distributed in a nanocrystal ensemble and therefore also in a sample. The delay is believed to characterize the migration of oxygen through the ZnS layer at grain boundaries and dislocations. ${ }^{27}$ Temporal and spatial random photooxidation and blue shift of a sparse enough subset of quantum dots into a spectrally confined detection channel forms the basis of our solution for GSDIM of densely labeled structures.

Figure $2 \mathrm{~B}$ exemplifies the proposed image acquisition scheme for three red-emitting quantum dots located within the diffraction limited volume (DLV). With nonchanging identical labels, GSDIM of normally labeled samples is compromised due to the brief off times. Stemming from several emitters with nearly identical emission, the blinking traces do not allow attribution to individual emitters. How- 


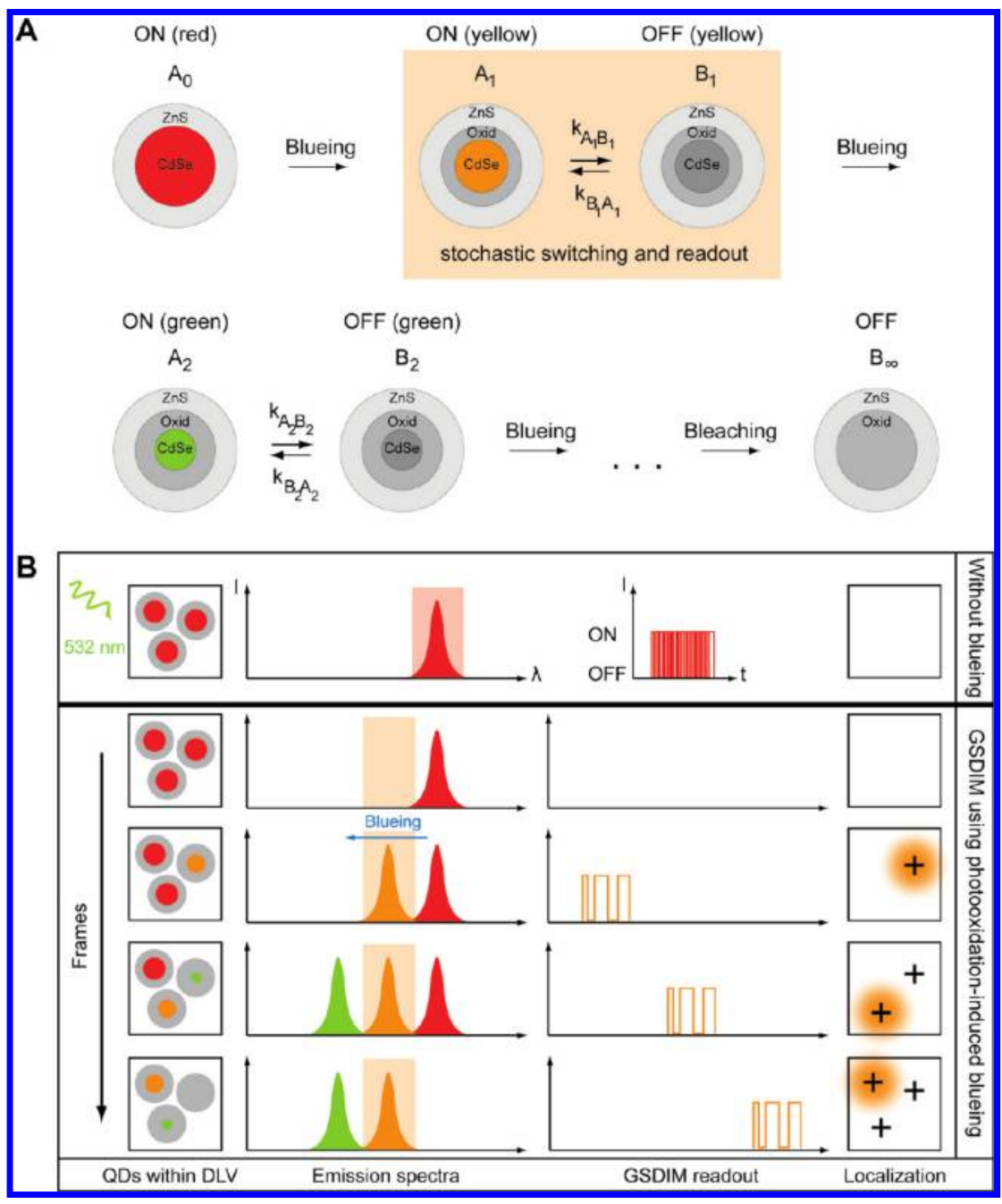

FIGURE 2. (A) Blue shift of emission exemplified for red-emitting quantum dots. Under continuous illumination, the size of the CdSe core decreases by photooxidation, continuously shifting the emission to the blue, ideally across the whole spectrum. Switching between fluorescence on and off states is apparent during the entire course of blueing until the core is bleached. The on and off state of a blue-shifted quantum dot marker is used for stochastic switching and readout. (B) Blue shift enables GSDIM. Without blueing, the signal from quantum dots overlaps within a diffraction limited volume (DLV) at a given time point due to rather short off periods. Disparate time periods between illumination and onset of blueing separate the fluorescence traces. Successive spectral translation of subsets of quantum dots into the blue-shifted detection window yields a diffraction unlimited image benefitting from the huge number of photons gained from a dot before bleaching or further blueing.

ever, by shifting the detection window toward shorter wavelengths, the traces of individual emitters become visible because the spatial density of blinking emitters is reduced.

Under steady laser illumination, a subset of native nanocrystals is stochastically photooxidized and blue shifted into the new detection window chosen such that all quantum dots are initially in the off state. Upon entering the detection window, the dots are localized. In the course of image acquisition, sparse subsets of quantum dot markers are successively blue shifted into the spectral detection window and contribute to the GSDIM image. The overall effective off time of the quantum dots therefore is much larger because it includes their native off time and the time the quantum dot is outside the spectral detection window or is eventually bleached. Image acquisition is stopped when more than $90 \%$ of all native nanocrystal markers have been shifted and localized.

It should be noted that even in the blue-shifted state the dot emits a large number of photons before being bleached or further blue shifted. The large photon number is a major advantage of GSDIM imaging over other stochastic approaches. Adding all photons emitted by a single quantum dot during the entire measurement yields an effective localization precision close to the quantum dot core diameter of about $4 \mathrm{~nm}$. Moreover, due to the large number of detected photons, most emitters are localized with high precision. This is in contrast to the virtually all stochastic superresolution (PALM, STORM, or GSDIM) applications reported so far, which by using organic fluorophores or 


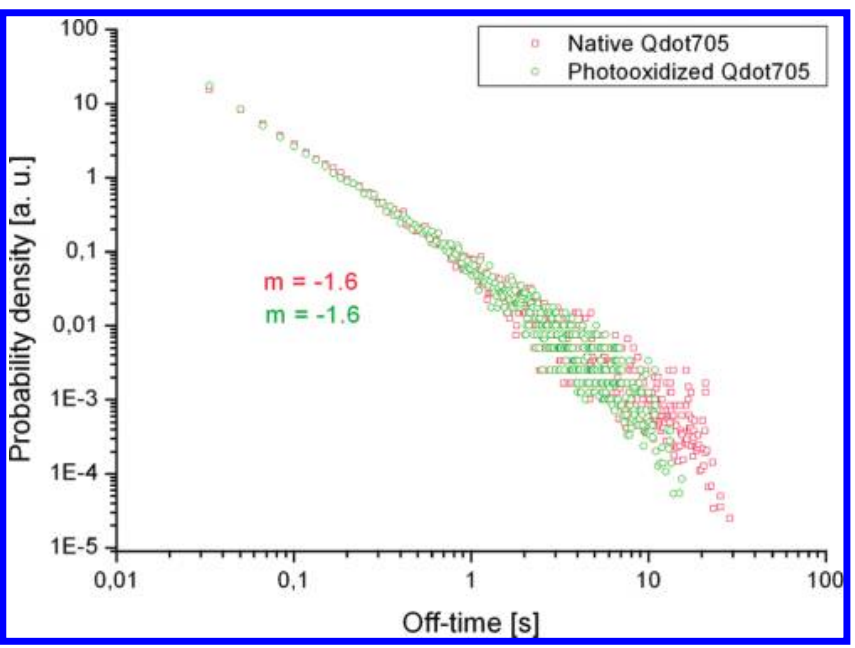

FIGURE 3. Photooxidation of the quantum dot core does not affect off-time duration. The off-time probability distributions after photoinduced blueing (green circles) at $19 \mathrm{~kW} \mathrm{~cm}^{-2}$ has been evaluated (according to Kuno et al. ${ }^{33}$ ) from $\sim 200$ quantum dot time traces and compared to their native blinking behavior before blueing (red squares). Matching power law exponents exhibit no off-time elongation in blue-shifted quantum dots.

fluorescent proteins discard the labels that, lacking sufficient brightness, cannot be localized precisely.

It is also worth mentioning that in contrast to approaches relying on separation of spectrally distinct organic dyes within a densely labeled sample, the quantum dot markers applied here are initially of the same type; they exhibit identical chemical, photochemical, and photophysical properties. Stochastic photooxidation during the measurement finally opens the door to a continuous space of separable states and enables diffraction-unlimited imaging.

The capability of this imaging scheme is demonstrated in immunofluorescence images of the microtubule network in mammalian PtK2 cells which has been labeled with commercially available quantum dots (Qdot 705 goat $F\left(a^{\prime} b^{\prime}\right) 2$ antimouse IgG conjugate $(\mathrm{H}+\mathrm{L})$, Invitrogen, Carlsbad, $\mathrm{CA}$ ). The conventional epifluorescence image (Figure 4A) of the filamentous cytoskeleton structures of microtubules is contrasted with the resulting GSDIM image (Figure 4B) resolving individual filaments. To determine the resolution attainable under the applied conditions, single isolated spots in the cell were identified, most likely representing individual nonspecifically bound quantum dot markers. With a $5 \mathrm{~nm}$ pixel size (fulfilling the Nyquist criterion), a Gaussian fit to the line profile across the localization cluster of these particles exhibits a fwhm of $12 \mathrm{~nm}$, giving a measure of the image resolution inside the cell (Figure 4G1). The average photon rate of a single quantum dot exceeds 1000 per frame, giving a localization accuracy of $\sim 10 \mathrm{~nm}$, detection noise considered. The measured effective resolution of $12 \mathrm{~nm}$ is thus in agreement with the theoretical expectation. By the same token, the many emitted photons afford short acquisition times. The apparent width of an individual microtubule filament in the GSDIM image is determined from its lateral

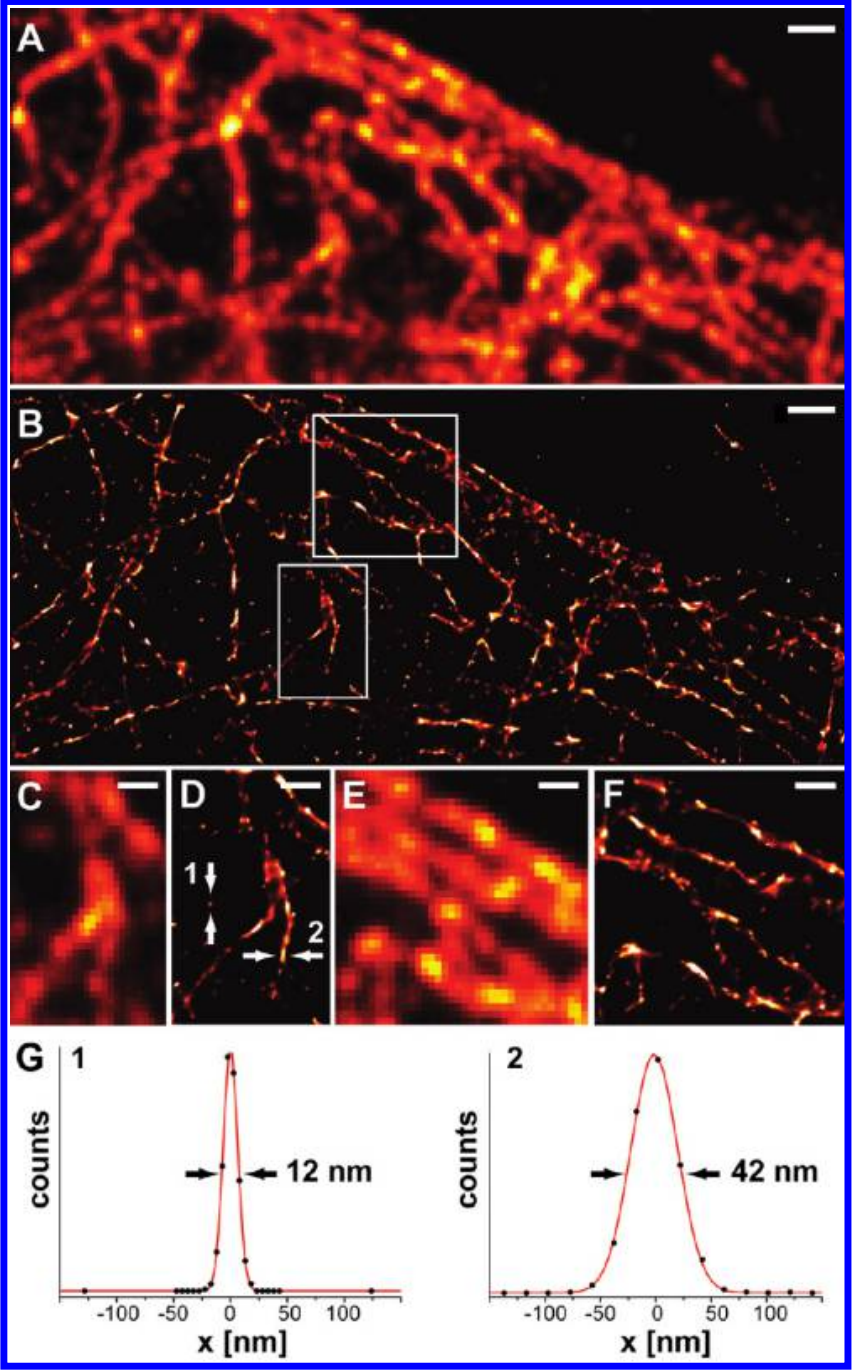

FIGURE 4. Quantum dot markers enable far-field optical nanoscopy by GSDIM. (A) Conventional epifluorescence image of the microtubular network in a fixed mammalian cell generated by taking the maximum projection of 1000 camera frames recording native quantum dots. The low excitation intensity $\left(15 \mathrm{~W} \mathrm{~cm}^{-2}\right)$ kept the initial blueing negligibly small. (B) GSDIM enabled by blueing. With $19 \mathrm{~kW} \mathrm{~cm}{ }^{-2}$ illumination by a diode laser emitting at $532 \mathrm{~nm}$, a set of 120000 frames with $15 \mathrm{~ms}$ frame time has been recorded. The many frames ensure that $>90 \%$ of all markers are shifted into the detection channel ranging from 538 to $645 \mathrm{~nm}$ and contribute to the image having a pixel size of $20 \mathrm{~nm}$. Scale bars: $1 \mu \mathrm{m}$. Regions in boxes are magnified in (D) and (F). The corresponding epifluorescence images are displayed in (C) and (E). The line profiles of a single quantum dot marker and a single microtubule strand are shown in (G1) and (G2), respectively. Black dots represent the data points of the GSDIM image, while the red line is the corresponding Gaussian fit. Scale bars: $500 \mathrm{~nm}$.

cross-sectional profile shown in Figure 4G2. Derived from a Gaussian fit to the profile, the fwhm of quantum dot stained microtubules is $42 \mathrm{~nm}$. Considering the $\sim 25 \mathrm{~nm}$ diameter of bare microtubule strands plus the primary and secondary antibodies having an overall size of $5-10 \mathrm{~nm}$ on either side, the fwhm matches the above diameter estimate.

The success of stochastic superresolution approaches in the past few years ${ }^{34}$ is, among others, due to the 


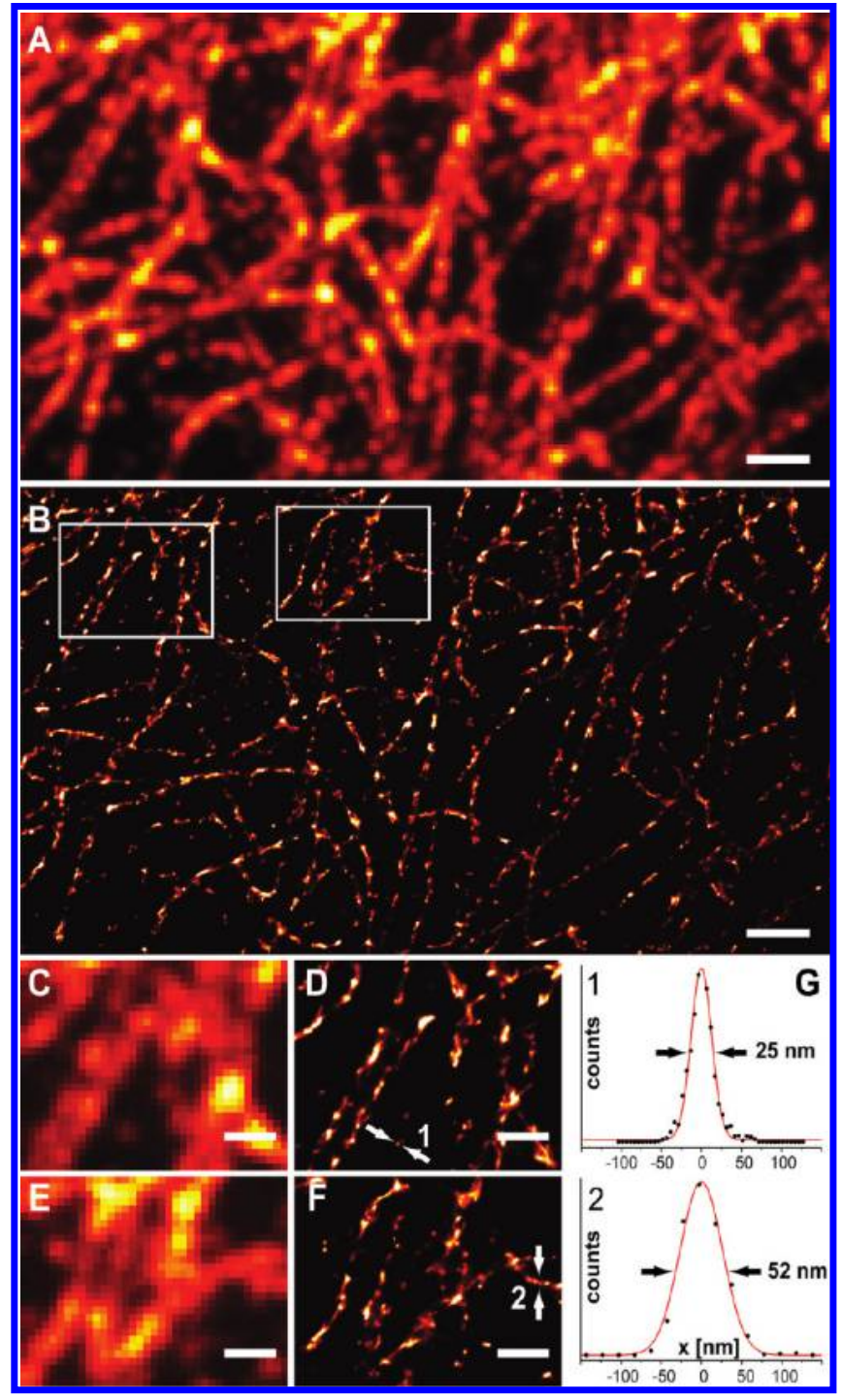

FIGURE 5. Webcam-GSDIM of cellular network. Spectral blueing of quantum dot markers enables the use of uncooled CCD cameras for detection, such as webcams, yielding GSDIM images with nanoscale resolution. (A) Epifluorescence image of microtubule filaments in a fixed mammalian cell. (B) GSDIM image enabled by blueing of quantum dot fluorescence employing a webcam. Upon illumination with a $532 \mathrm{~nm}$ diode laser, a set of 60000 frames was recorded with a readout rate of 30 frames/s. Scale bars: $2 \mu \mathrm{m}$. The pixel size of 20 $\mathrm{nm}$ was decreased to $5 \mathrm{~nm}$ for resolution estimates. Boxed image regions magnified in (D) and (F) exhibit the resolution increase over the diffraction limited counterparts in (C) and (E). Profiles cutting through a single quantum dot marker and a single microtubule strand are displayed in (G1) and (G2), respectively. The black dots show the data points of the line profile; the red line is the corresponding Gaussian fit. Scale bars: $1 \mu \mathrm{m}$.

technical simplicity of the setup. The component partially inhibiting its wider use is the mandatory sensitive electron multiplying EM CCD camera which has so far been the only array detector sensitive enough for fast readout of single emitters. Our concept of quantum dot labeling now enables the use of much less sensitive detectors. The emitted signal is so bright that the thermally induced noise of conventional CCDs is readily exceeded. In Figure 5, the same microtubules sample measured by the EM CCD camera was now imaged using a webcam (Philips SPC900NC, Koninklijke Philips Electronics N.V., Amsterdam, The Netherlands). Compared to the epifluorescence image, the resolution enhancement of webcam-GSDIM imaging is striking. Two regions of the GSDIM image have exemplarily been highlighted to display the resolved individual microtubule filaments. Quantitatively, the resolution potential is determined from the width of single, isolated spots in the image background; it amounts to $\sim 25$ $\mathrm{nm}$. Neither the relatively high readout noise nor the internal compression of recorded images by the webcam obstructs attaining resolution at the nanoscale. The subdiffraction webcam image is qualitatively and quantitatively comparable to the GSDIM image acquired by an EM CCD camera. It is also worthwhile noting that GSDIM is stochastic not only in space but also in time. GSDIM readout works with comparatively slow frame rates, because the blinking need not to be adjusted to the camera integration time.

Finally, it should be noted that the emission behavior of spherical quantum dots differs from most organic single dipole emitters. ${ }^{35,36}$ Their largely isotropic emission makes them less susceptible for localization errors caused by anisotropy effects.

In conclusion, we have shown that some of the unique properties of semiconductor quantum dots enable GSDIM nanoscopy with resolution down to about the size of a single dot. The challenge initially posed by the short dark periods has been met by successive blueing in exciton emission, enabling temporal and spatial separation of densely quantum dot labeled biological structures at the nanoscale. In the future, quantum dot off periods may be extended by nanocrystal modification or by addition of appropriate electron acceptors or donors to the sample. EM CCD cameras are no longer required. Nanoscale resolution has become possible using webcams. In fact, far-field fluorescence nanoscopy is now drastically simplified in terms of the required hardware. With quantum dots, any wide-field microscope retrofitted with a low-power diode laser and a low-priced webcam can be converted into an optical nanoscope. A powerful variant of fluorescence nanoscale imaging has thus become readily accessible, fueling the ongoing revolution in far-field optical resolution.

Acknowledgment. We thank our colleagues R. Medda for preparing the samples and E. Rittweger and P. Bingen for valuable discussions and critical reading.

Supporting Information Available. Details of the sample preparation and the optical setup. This material is available free of charge via the Internet at http://pubs.acs.org/.

\section{REFERENCES AND NOTES}

(1) Abbe, E. Arch. Mikrosk. Anat. 1873, 9, 413-468.

(2) Hell, S. W.; Wichmann, J. Opt. Lett. 1994, 19 (11), 780-782. 
(3) Hell, S. W.; Kroug, M. Appl. Phys. B: Lasers Opt. 1995, 60, 495497

(4) Betzig, E.; Patterson, G. H.; Sougrat, R.; Lindwasser, O. W Olenych, S.; Bonifacino, J. S.; Davidson, M. W.; LippincottSchwartz, J.; Hess, H. F. Science 2006, 313 (5793), 1642-1645.

(5) Rust, M. J.; Bates, M.; Zhuang, X. W. Nat. Methods 2006, 3, 793 795.

(6) Hess, S. T.; Girirajan, T. P. K.; Mason, M. D. Biophys. I. 2006, 91 (11), 4258-4272.

(7) Hell, S. W. Science 2007, 316 (5828), 1153-1158.

(8) Heisenberg, W., The physical principles of the quantum theory; Chicago University Press: Chicago, IL, 1930

(9) Thompson, R. E.; Larson, D. R.; Webb, W. W. Biophys. I. 2002, 82, 2775-2783

(10) Fölling, J.; Bossi, M.; Bock, H.; Medda, R.; Wurm, C. A.; Hein, B.; Jakobs, S.; Eggeling, C.; Hell, S. W. Nat. Methods 2008, 5, 943-945.

(11) Heilemann, M.; van de Linde, S.; Schuttpelz, M.; Kasper, R.; Seefeldt, B.; Mukherjee, A.; Tinnefeld, P.; Sauer, M. Angew. Chem. Int. Ed. 2008, 47, 6172-6176

(12) Egner, A.; Geisler, C.; von Middendorff, C.; Bock, H.; Wenzel, D. Medda, R.; Andresen, M.; Stiel, A.-C.; Jakobs, S.; Eggeling, C.; Schoenle, A.; Hell, S. W. Biophys. I. 2007, 93, 3285-3290.

(13) Bruchez, M.; Moronne, M.; Gin, P.; Weiss, S.; Alivisatos, A. P Science 1998, 281, 2013-2016.

(14) Resch-Genger, U.; Grabolle, M.; Cavaliere-Jaricot, S.; Nitschke, R.; Nann, T. Nat. Methods 2008, 5, 763-775.

(15) Irvine, S. E.; Staudt, T.; Rittweger, E.; Engelhardt, J.; Hell, S. W Angew. Chem. Int. Ed. 2008, 47 (14), 2685-2688.

(16) Moerner, W. E.; Orrit, M. Science 1999, 283, 1670-1676.

(17) Nirmal, M.; Dabbousi, B. O.; Bawendi, M. G.; Macklin, J. J.; Trautman, J. K.; Harris, T. D.; Brus, L. Nature 1996, 383, $802-$ 804.

(18) Mahler, B.; Spinicelli, P.; Buil, S.; Quelin, X.; Hermier, J. P.; Dubertret, B. Nat. Mater. 2008, 7, 659-664

(19) Wang, X.; Ren, X.; Kahen, K.; Hahn, M. A.; Reajeswaran, M.; Maccagnano-Zacher, S.; Silcox, J.; Cragg, G. E.; Efros, A. L.; Krauss, T. D. Nature 2009, 459, 686-689.
(20) Hohng, S.; Ha, T. I. Am. Chem. Soc. 2003, 126, 1324-1325.

(21) Chen, Y.; Vela, J.; Htoon, H.; Casson, J. L.; Werder, D. J.; Bussian, D. A.; Klimov, V. I.; Hollingsworth, J. A. I. Am. Chem. Soc. 2008, 130, 5026-5027.

(22) Fomenko, V.; Nesbitt, D. I. Nano Lett. 2008, 8, 287-293

(23) Dertinger, T.; Colyer, R.; Iyer, G.; Weiss, S.; Enderlein, J. Proc. Natl. Acad. Sci. U.S.A. 2009, 106, 22287-22292.

(24) Lidke, K. A.; Rieger, B.; Jovin, T. M.; Heintzmann, R. Opt. Express 2005, 13 (18), 7052-7062.

(25) Shimizu, K. T.; Neuhauser, R. G.; Leatherdale, C. A.; Empedocles, S. A.; Woo, W. K.; Bawendi, M. G. Phvs. Rev. B 2001, 63, 205316.

(26) Cordero, S. R.; Carson, P. J.; Estabrook, R. A.; Strouse, G. F.; Buratto, S. K. I. Phys. Chem. B 2000, 104, 12137-12142.

(27) van Sark, W. G. J. H. M.; Frederix, P. L. T. M.; den Heuvel, D. J. V.; Gerritsen, H. C. J.; Bol, A. A.; van Lingen, J. N. J.; de Mello Donegá, C.; Meijerink, A. J. Phys. Chem. B 2001, 105, 8281-8284.

(28) van Sark, W. G. J. H. M.; Frederix, P. L. T. M.; Bol, A. A.; Gerritsen, H. C.; Meijerink, A. ChemPhysChem 2002, 3 (10), 871-879.

(29) Empedocles, S. A.; Bawendi, M. G. Science 1997, 278, 2114-2117.

(30) Seufert, J.; Obert, M.; Scheibner, M.; Gippius, N. A.; Bacher, G.; Forchel, A.; Passow, T.; Leonardi, K.; Hommel, D. Appl. Phvs. Lett. 2001, 79, 1033-1035

(31) Katari, J. E. B.; Colvin, V. L.; Alivisatos, A. P. J.Phys. Chem. 1994, 98, 4109-4117.

(32) Nazzal, A. Y.; Wang, X. Y.; Qu, L. H.; Yu, W.; Wang, Y. J.; Peng, X. G.; Xiao, M. J. I. Phvs. Chem. B 2004, 108, 5507-5515.

(33) Kuno, M.; Fromm, D. P.; Hamann, H. F.; Gallagher, A.; Nesbitt, D. J. L. Chem. Phvs. 2001, 115, 1028-1040.

(34) Hell, S. W. Nat. Methods 2009, 6 (1), 24-32.

(35) Patra, D.; Gregor, I.; Enderlein, J. Appl. Phys. Lett. 2005, 87, 101103

(36) Chung, I.; Shimizu, K. T.; Bawendi, M. G. Proc. Natl. Acad. Sci. U.S.A. 2003, 100 (2), 405-408 